\title{
MicroRNA-210 expression during childbirth and postpartum as a potential biomarker of acute fetal hypoxia
}

\author{
Barbara Vonkova ${ }^{\mathrm{a}, \mathrm{b}, \mathrm{d}}$, Ivona Blahakova ${ }^{\mathrm{a}, \mathrm{b}}$, Lukas Hrubanc, Petr Jankuc, Sarka Pospisilova ${ }^{\mathrm{a}, \mathrm{b}}$
}

\begin{abstract}
Objective. To explore whether miR-210 expression can be used as a diagnostic and prognostic marker in acute fetal hypoxia.

Methods. Whole blood samples of 29 women and their fetuses without hypoxia and 24 women and their fetuses with hypoxia were analysed in this study. Reverse transcription and quantitative real-time PCR were used to measure the expression of miR-210. Expression level differences between the control and hypoxic group in labour time and postpartum change fold were analyzed by standard statistical tests.

Results. We confirmed that miR-210 is significantly more upregulated in fetal blood with acute hypoxia when compared to maternal blood $(P<0.001)$. Furthermore, there was significant up-regulation in miR-210 level in the hypoxic group when compared to the control non-hypoxic group $(P<0.05)$ in both maternal and fetal blood. Our results did not confirm a significant difference in postpartum miR-210 clearance level $2 \mathrm{~h}, 8 \mathrm{~h}, 24 \mathrm{~h}$ or $48 \mathrm{~h}$ after labour.

Conclusions. Our study confirmed miR-210 upregulation in the blood of pregnant women with acute fetal hypoxia at the time of labour compared to pregnant women without acute fetal hypoxia. Additional investigation should be done to determine miR-210 clearance and the possibility of using miR-210 as a diagnostic and prognostic marker.
\end{abstract}

Key words: microRNA-210, acute fetal hypoxia, postpartum, clearance of miR-210

Received: April 20, 2018; Revised: October 10, 2018; Accepted: December 4, 2018; Available online: December 18, 2018 https://doi.org/10.5507/bp.2018.075

(c) 2019 The Authors. This is an open access article licensed under the Creative Commons Attribution License

(https://creativecommons.org/licenses/by/4.0/).

${ }^{a}$ Center of Molecular Medicine, CEITEC - Central European Institute of Technology, Masaryk University, Brno, Czech Republic ${ }^{b}$ Center of Molecular Biology and Gene Therapy, Department of Internal Medicine - Hematology and Oncology, University Hospital Brno, Czech Republic

'Department of Gynaecology and Obstetrics, The University Hospital Brno, Czech Republic

${ }^{d}$ Faculty of Medicine, Masaryk University, Brno, Czech Republic

Corresponding author:Sarka Pospisolova, e-mail:pospisilova.sarka@fnbrno.cz

\section{INTRODUCTION}

Even though the fetus has its own natural defence mechanism to tackle the oxygen insufficiency during delivery, in some cases, only timely intervention can prevent adverse consequences ${ }^{1}$. Hypoxia, prevalent in the range of $0.6 \%$ (ref. $^{2}$ ) to $3.5 \%$ (ref. $^{3}$ ), is considered to be the third most common cause of newborn deaths ${ }^{4}$ in developed countries. In addition to fetal or newborn death, hypoxic ischemic encephalopathy (HIE) is the immediate consequence of hypoxia in the newborn. The long-term consequences being cerebral palsy. The overall prevalence of cerebral palsy in developed countries remains stable at around $2 \%$, regardless of a variety of reduction efforts 5 .

The fetal stethoscope - introduced by Pinard in 1876 - was replaced in late the 1960's by cardiotocography (CTG) - to date it is the standard method of intrapartum hypoxia detection. It did not, however, bring the expected improvements in delivery outcomes compared to the previously used intermittent auscultation ${ }^{6}$ and, moreover, continuous CTG is one of the main suspects for the increased rate in casarean sections for objective reasons ${ }^{6}$. The cause of CTG's unsatisfactory results is primarily considered to be the high level of inter- and even intra-ob- server disagreement on evaluation ${ }^{7-9}$. The problems with CTG evaluation are not only at the theoretical level, with substantial medico-legal consequences being repeatedly reported ${ }^{10-11}$. Nevertheless, CTG remains the main instrument to directly assess the well-being of a fetus during delivery. Adjunct methods try to enhance the specificity of CTG interpretation: e.g. fetal scalp blood sampling (taken to measure the fetal scalp capillary $\mathrm{pH}$ ); or measuring the fetal ECG (STAN). All methods are used only in addition to CTG and rely on correct CTG interpretation (FIGO consensus guidelines 2015) (ref. ${ }^{5}$ ).

Confirming the diagnosis of intrapartum hypoxia of the fetus with metabolic acidosis is currently based exclusively on determining $\mathrm{pH}$ and base deficit in the extracellular fluid (BDefc) from the umbilical artery, or lactate levels, estimated immediately after delivery ${ }^{5,12}$. Even this method is not perfect and can be used only postpartum. At present, there is no method available to accurately detect fetal hypoxia during labour.

It was shown that low levels of oxygen change the expression of a many genes. This allows the cells to run adaptive processes that involve changes in cell division, survival, or differentiation. The reaction to hypoxia also occurs at the post-translational level, with increased ex- 
pression of some miRNAs. The major miRNA responding to low oxygen levels is miR-210, whose increased expression has been described in many studies ${ }^{13-14}$. MiRNAs in the maternal circulation are mostly derived from the placenta and are present from early pregnancy, increase across gestation and disappear after delivery ${ }^{15}$. The exact mechanism by which miRNAs enter the maternal circulation is largely unknown, but they may be released from the syncytiotrophoblast by exosomes ${ }^{16}$. More than $90 \mathrm{mi}-$ croRNAs that are induced by hypoxia and modulate the cellular response to hypoxia have been identified ${ }^{17-18}$.

Whitehead ${ }^{19}$ concluded that the number of hypoxicinduced miRNAs increase across labour and the amount of hypoxia-induced miRNAs correlate with the degree of in-utero hypoxia. Also, there was a 3-fold increase in combined miR-21 and miR-20b expression in the fetuses with high lactate.

The aim was to identify a more accurate marker for the ongoing intrauterine fetal distress that could be determined before delivery. Such a marker could refine fetal hypoxia detection, which is currently based exclusively on CTG. Linking these two methods could significantly increase the sensitivity and specificity to detect severe fetal hypoxia, reducing the percentage of unnecessary surgical interventions.

\section{METHODS AND MATERIALS}

\section{Study participants}

A total of 53 pregnant women were recruited from the Department of Obstetrics and Gynaecology at Brno University Hospital, the Czech Republic between August 2016 and May 2017. Over 6000 births per year take place in this department. All women provided written informed consent and ethical approval has been obtained. Inclusion criteria were as follows: non-smoker, no diabetes mellitus, 38-40 week of pregnancy, the age of mother 18-36. The clinical criteria were: single birth only, vaginal delivery (the caesarean section was excluded). In the control group (29 participants), women with physiological pregnancies and births were selected, while women with acute fetal hypoxia were included in the hypoxic group (24 participants) (Table 1). Women with chronic hypoxia, preeclampsia and fetal growth restriction were omitted.

\section{Sample collection}

Samples were collected at the time of delivery from both the control and the hypoxic group. Thenceforward after $2 \mathrm{~h}, 8 \mathrm{~h}, 24 \mathrm{~h}$, and $48 \mathrm{~h}$ after delivery in the hypoxic group to catch immediate changes in miR-210 levels. In the control group, the samples were collected $2 \mathrm{~h}$ and $24 \mathrm{~h}$ after delivery. At every time interval, the maternal peripheral whole blood sample $(2.5 \mathrm{~mL})$ was collected, and blood samples were taken from the newborns at the time of delivery from the fetal umbilical artery. Samples for the hypoxic group were selected mainly based on pathological CTG at the end of labor and after postpartum $\mathrm{pH}(<7.2)$ and base excess (BE>12 mmol/L) confirmation taken immediately after labor.
Table 1. Study cohort characterization.

\begin{tabular}{lccc}
\hline & & Hypoxic & Control \\
\hline Gender of child & Boy & 11 & 19 \\
& Girl & 13 & 10 \\
Mother age & $18-30$ & 13 & 11 \\
\multirow{3}{*}{ Gestation age } & $31-36$ & 11 & 18 \\
& 38 & 4 & 6 \\
& 39 & 6 & 5 \\
& 40 & 8 & 9 \\
$\mathrm{pH}$ & 41 & 2 & 8 \\
& 42 & 4 & 1 \\
& $7.19-7.15$ & 11 & \\
& $7.14-7.10$ & 6 & \\
\hline
\end{tabular}

Specimen processing and quantitative RT-PCR assay

All blood samples were collected into PAXgene Blood RNA tubes (Preanalytix). After $2 \mathrm{~h}$ at room temperature, the total RNA was isolated using the PAXgene Blood miRNA Kit (Preanalytix) according to the manufacturer's instructions. Then total RNA concentration and quality were measured using a NanoDrop ND-1000 Spectrophotometer. RNA was reversely transcribed with TaqMan MicroRNA Reverse Transcription Kit (ThermoFisher Scientific) and Reverse Transcription Master Mix (Generi Biotech), according to the manufacturer's protocols.

MiR-210, RNU6B, and RNU48 as an internal control were determined by quantitative real-time PCR with TaqMan MicroRNA Assays and Quantitative PCR was performed with the standard cycling conditions.

\section{Statistical analysis}

The comparative CT $\left(2^{-\Delta \mathrm{CT}}\right)$ method was used to normalize all results against the mean expression of internal controls (RNU6B and RNU48). Then samples obtained in time after labour were compared by fold change expression. All data were evaluated in SDS v2.4 (Sequence Detection System) and statistically analyzed using GraphPad Prism 5.0. A significant difference was defined as $\mathrm{P}<0.05$.

\section{RESULTS}

\section{Hypoxic group characterization}

The $\mathrm{pH}$ level was taken immediately after labor from the umbilical artery. Based on this result and according to the CTG, the hypoxic group was selected. Also, the BMI (Body Mass Index) of the pregnant women was monitored at the time of admission to the hospital. The minimal $\mathrm{pH}$ level was 6.8 and the median was 7.12. The maximal BMI was 45.9 and median was 27.7 (Table 1)

Expression of miR-210 in acute fetal hypoxia during labour After confirming miR-210 presence in fetal and mater- 
(a) control group

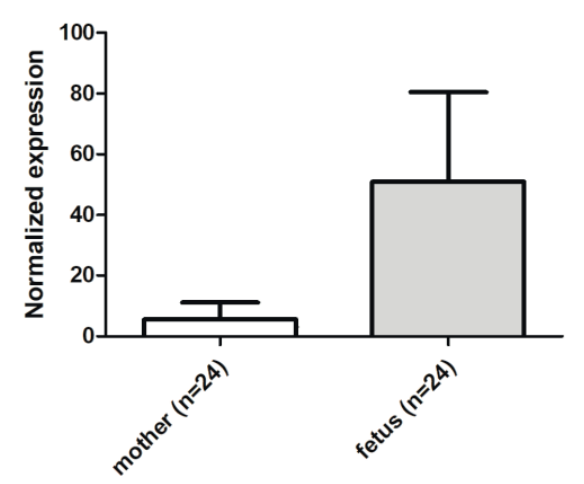

(b) hypoxic group

$P<0.001$

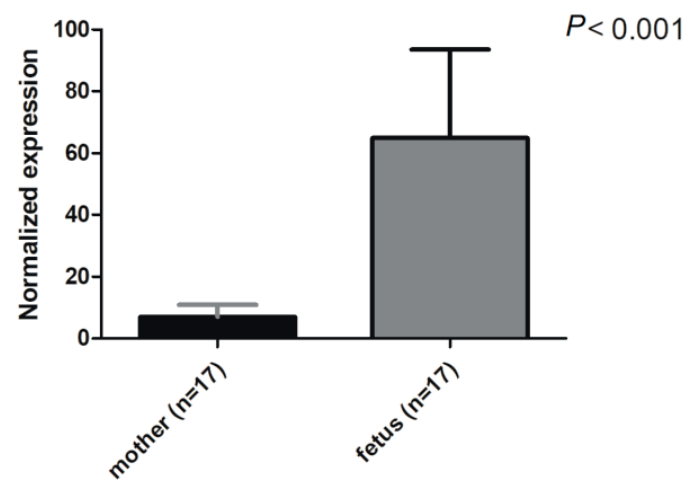

Fig. 1. Bar chart showing relative expression of miR-210 in maternal and fetal blood during labour in (a) control group and (b) hypoxic group. Relative expression of miR-210 is significantly higher in the fetal blood compared with the maternal blood $(P<0.001)$.

(a) Fetal expression levels of miR-210 in control and hypoxic group

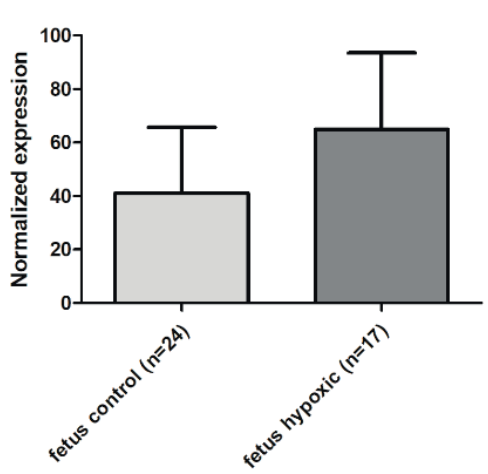

(b) Mother expression levels of miR-210 in control and hypoxic group

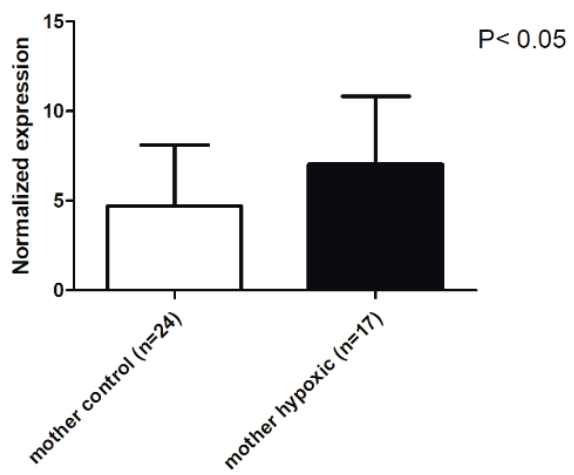

Fig. 2. Bar chart showing relative expression of miR-210 in control vs. hypoxic group during the labour. Samples of fetal and maternal blood were compared. Relative expression of miR-210 is significantly higher in hypoxic group compared with control group $(P<0.05)$ in both fetal and maternal blood. (a) levels of miR-210 in fetal blood samples, (b) levels of miR-210 in maternal blood samples.

(a) Control group

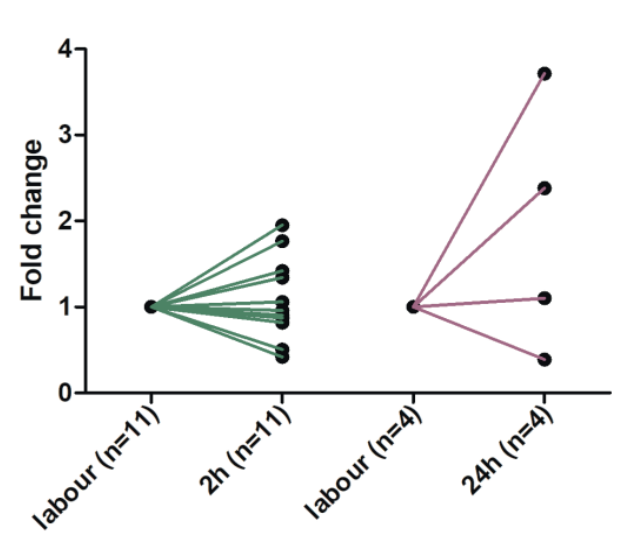

(b) Hypoxia group

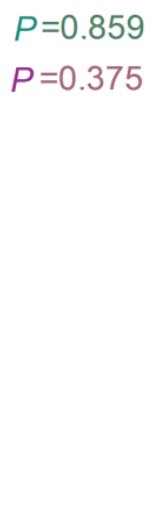

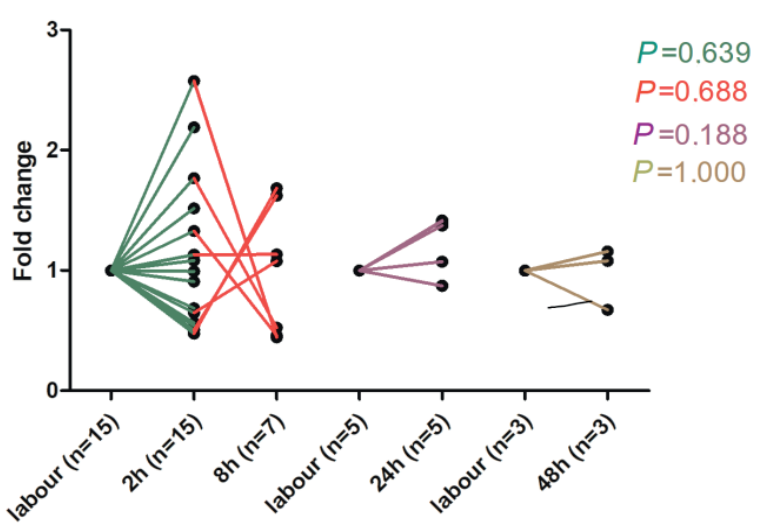

Fig. 3. Fold change showing changes of expression of miR-210 in maternal blood after the labour in time. (a) control group, samples were analysed after $2 \mathrm{~h}$ and $24 \mathrm{~h}$ after the labour. There is no significant change of miR-210 expression after the labour. (b) hypoxic group, samples were analysed after $2 \mathrm{~h}, 8 \mathrm{~h}, 24 \mathrm{~h}$ and $48 \mathrm{~h}$ after labour. There is no significant change of miR-210 expression after the labour. 
nal blood samples at the time of delivery, we compared miR-210 levels in the fetal and maternal blood in both groups (control and hypoxic).

The results were created from 24 mothers from the control group and 17 mothers from the hypoxic group. Other samples were not included due to insufficient mother or child or both blood collection in the time of delivery (this involved 5 mothers from the control group and 7 mothers from the hypoxic group). There was a significantly higher expression in fetal blood samples in comparison with maternal blood samples in both the control (median 4.17 vs. 44.90 ; $P<0.001$; Fig. 1a) and hypoxic group (median 6.34 vs. 54.21 ; $P<0.001$; Fig. 1b)

Subsequently, we compared miR-210 levels in the hypoxic and control group in both maternal and fetal blood. We confirmed a significantly higher miR-210 expression in the hypoxic group than the control group (fetal expression in control group 44.90 vs. 54.21 in the hypoxic group; $P<0.05$; Fig. 2a and median in maternal expression in the control group 4.17 vs. 6.34 in the hypoxic group; $P<0.05$; Fig. 2b).

\section{Clearance of miR-210 after labour in maternal blood}

For clinical use, it is essential to know the dynamics of blood marker levels over time.

In this study, we analyzed miR-210 expression in the control and hypoxic groups at specific time intervals (see sample collection). In the control group, we did not find any significant miR-210 level changes either $2 \mathrm{~h}$ or $24 \mathrm{~h}$ after labour (median in labour 2.48 vs. 1.82 after $2 \mathrm{~h}$; $P=0.86$ and median after $24 \mathrm{~h} 7.59 ; P=0.375$; Fig. $3 \mathrm{a}$ ). We then analysed miR-210 levels in the hypoxic group and surprisingly there was also no difference in the level of expression between the time during labour (median 5.01) and $2 \mathrm{~h}$ after labour (median 6.24; $P=0.639$ ), $8 \mathrm{~h}$ after labour (median 3.56; $P=0.688$ ), 24 h (median 9.91; $P=0.188$ ) or $48 \mathrm{~h}$ after labour (median $4.167 ; P=1.00$; Fig. $3 b)$.

\section{DISCUSSION}

The supply of oxygen to the fetus by the placenta is a prerequisite for the proper growth, development and survival of the fetus. Acute fetal hypoxia during labour and poor function of the placenta during pregnancy leading to chronic fetal hypoxia are the most common causes of neonatal mortality and persistent, especially neurological consequences ${ }^{20}$. The occurrence of miR-210 expression associated with hypoxia has been demonstrated in some pathological conditions in pregnancy in association with trophoblastic hypoxia or the defective development of the placenta and its function. Preeclampsia and intrauterine growth retardation are among these conditions in pregnancy. However, an increase in miR-210 expression is observed in a number of physiological conditions as well as in the case of stress-induced hypoxia in malignan$\operatorname{cies}^{21-25}$ In our study, detectable miR-210 expression was observed in a group of pregnant women with confirmed fetal hypoxia, as well as in non-hypoxic pregnant women in the control group. The incidence in both groups suggests the key presence of miR-210 at the time of delivery, in addition to many hemodynamic changes, also to limit placental blood flow due to vasoconstriction caused by numerous contractions of the myometrium. Furthermore, in this study, a significantly higher miR-210 expression was found in the fetal blood compared to maternal blood at birth $(P<0.001)$. This difference is a reflection in the increased need for miR-210 in the baby's blood to control inadequate fetal oxygen supply during childbirth. However, the reason for increasing the expression of miR210 present in the mother's blood is still questionable, and further tests will be needed to verify its origin. Assuming that part of the miR-210 detected in the mother's blood is of fetal origin, and thus reflects the potential occurrence of hypoxia in a child, miR-210 detection can be used to monitor the status of the fetus before and during childbirth ${ }^{19}$. To verify the possibility of monitoring fetal hypoxia based on determining miR-210 expression, we compared the change in this expression between the control group and the hypoxic fetal group, both in the mother's blood and in the fetal blood. Normalization to the endogenous control of RNU48 showed a significantly higher miR-210 expression in the fetus-hypoxic group than the control group $(P<0.05)$, both in the mother's blood and in the blood of the child. This result confirmed a possible association between miR-210 expression and a child's pathological condition that is detectable from the mother's blood. Our confirmed increase in miR-210 expression in the hypoxic fetal group is consistent with the literature showing increased expression in both fetal hypoxia, intrauterine growth fetal restriction ${ }^{19}$ and other pathological conditions in pregnancy ${ }^{26-27}$.

Diagnosis of acute fetal hypoxia during childbirth could be a very effective tool to prevent the development of long-term consequences or death. Detection of miR-210 expression from mother blood appears to be a suitable approach for this diagnosis. However, the limitations of today's technological equipment mean that there is not yet possible to use it as a bed-side test. Thus, the detection of miR-210 expression is only a demonstration of future options for the detection of acute fetal hypoxia and other perinatal pathologies during childbirth.

At the beginning of this study, sampling was provided according to the Whitehead study ${ }^{19}$ at the time of the first contractions and the first delivery period. Due to the complicated determination of these prenatal intervals and the inability to identify acute fetal hypoxia before childbirth, only 5 samples were collected from mothers from the control group and only 2 samples from mothers from the hypoxic group. Because of the low number of samples thus collected, the sampling scheme was further optimized with a focus on the postpartum period.

Consequently, we continued to monitor the dynamics of miR-210 expression changes in post-partum delivery. A similar trend of change was assumed as for cffDNA, which is mainly used for diagnosing the fetal gene abnormalities from non-invasive maternal blood tests, as well 
as for miRNA specifically expressed in the placenta. In both cases, there is a significant decrease in the nucleic acid concentration observed after $24 \mathrm{~h}$ ( ref. $^{28-30}$ ). To closely monitor the changes in miR-210 expression after childbirth, we, therefore, chose $2 \mathrm{~h}, 8 \mathrm{~h}, 24 \mathrm{~h}$, and $48 \mathrm{~h}$ sampling times in the hypoxic group to capture changes in expression over a longer time interval and in the control group at $2 \mathrm{~h}$ postpartum and $24 \mathrm{~h}$ postpartum.

In this work, we did not see any statistically significant difference in miR-210 level at $2 \mathrm{~h}$ or $24 \mathrm{~h}$ postpartum in the control group. This outcome can be partly explained by the low level of miR-210 expression corresponding to the rate of the child's physiological response during childbirth, which therefore eliminates the need to lower this expression below the physiological limit.

In the hypoxic group, there was also no statistically significant decrease in miR-210 expression after $2 \mathrm{~h}, 8 \mathrm{~h}$, $24 \mathrm{~h}$ or $48 \mathrm{~h}$ postpartum compared to the delivery time. This result is in contrast with the decreasing trend in placental origin miRNA (ref. ${ }^{28-29}$ ), but the same result as in our study (no change of levels) confirms the monitoring of miR-210 expression at $8 \mathrm{~h}$ and $24 \mathrm{~h}$ after terminating the experimentally induced hypoxic status ${ }^{31}$. This result can be explained by the considerable miRNA stability in maternal and fetal blood where it is protected from the degradation processes by the vesicle's membrane in which it is located, or its binding to blood proteins ${ }^{16,32-33}$. This phenomenon is common to other miRNAs, whose expression is induced by trophoblast hypoxia in IUGR (ref. ${ }^{34}$ ).

One of the aims of this study was to verify observations of increased expression of miR-210 published by Whitehead ${ }^{19}$ on a cohort of 8 hypoxic patients and to extend this study group. We confirmed the observation on the cohort of 17 hypoxic samples. However, our results are still influenced by the limited number of samples taken at all time intervals from one patient to maintain the possibility of statistical paired testing. Other factors significantly affecting the expression analysis are the nonstandardized endogenous controls used to normalize the expression of placental origin genes ${ }^{3436}$. Further studies must be conducted to find out if and when there is a decrease of miR-210 after birth and thus allow the use of this diagnostic marker and in other pregnancies.

Our results could also be affected by the biological variability of the individual specimens, as the miR-210 expression is, among other things, associated with the BMI of the mother ${ }^{37}$. In conclusion, the miR-210 expression change at post-partum was not confirmed by our study, which is in line with previously reported results. It is also important to consider the impact of the severity of hypoxia (according to $\mathrm{pH}$ ) to miRNA expression and duration of hypoxia status (vary from minutes to hours in acute hypoxia cases and days or week in chronic hypoxia). However, our results did not observe a direct correlation between $\mathrm{pH}$ level and fetal hypoxia severity. After all, the results of this work were considerably limited by the number of input samples and the variable expression of the endogenous controls to which the expression of the selected indicators was normalized.
Acknowledgment: This research was supported by startup grant Sup 1/16 provided by The University Hospital Brno, Czech Republic. It was also supported by projects from the Faculty of Medicine, Masaryk University MUNI/A/0968/2017. This research was carried out under the project CEITEC 2020 (LQ1601) with financial support from the Ministry of Education, Youth and Sports of the Czech Republic under the National Sustainability Programme II. This work was also supported by the research grant TACR (TE02000058/2014-2019).

Author contributions: BV: conception and design, acquisition of data, data analysis and interpretation, drafting of the manuscript, statistical analysis; LH: conception and design, drafting of the manuscript, administrative technical and material support; IB: conception and design; data analysis and interpretation, drafting of the manuscript, obtaining funding; PJ, SP: critical revision of the manuscript for important intellectual content, obtaining funding.

Conflict of statement interest: The authors report no conflict of interest.

\section{REFERENCES}

1. Alfirevic Z, Devane D, Gyte GM, Cuthbert A. Continuous cardiotocography (CTG) as a form of electronic fetal monitoring (EFM) for fetal assessment during labour. Cochrane Database Syst Rev 2007;2:CD006066.

2. Heintz E, Brodtkorb TH, Nelson N, Levin LA. The long-term costeffectiveness of fetal monitoring during labour: a comparison of cardiotocography complemented with ST analysis versus cardiotocography alone. BJOG Int J Obstet Gynaecol 2008;115:1676-87.

3. Strachan BK, van Wijngaarden WJ, Sahota D, Chang A, James DK. Cardiotocography only versus cardiotocography plus PR-interval analysis in intrapartum surveillance: a randomised, multicentre trial. FECG Study Group. Lancet Lond Engl 2000;355:456-9.

4. d'Aloja E, Müller M, Paribello F, Demontis R, Faa A. Neonatal asphyxia and forensic medicine. J Matern Fetal Neonatal Med Off J Eur Assoc Perinat Med Fed Asia Ocean Perinat Soc Int Soc Perinat Obstet 2009;22(Suppl 3):54-6.

5. Visser GH, Ayres-de-Campos D and FIGO Intrapartum Fetal Monitoring Expert Consensus Panel. FIGO consensus guidelines on intrapartum fetal monitoring: Adjunctive technologies. Int J Gynaecol Obstet Off Organ Int Fed Gynaecol Obstet 2015;131:25-9.

6. Steer PJ. Has electronic fetal heart rate monitoring made a difference. Semin Fetal Neonatal Med 2008;13:2-7.

7. Blackwell SC, Grobman WA, Antoniewicz L, Hutchinson M, Gyamfi Bannerman C. Interobserver and intraobserver reliability of the NICHD 3-Tier Fetal Heart Rate Interpretation System Am J Obstet Gynecol 2011;205:378.e1-5.

8. Ayres-de-Campos D, Bernardes J, FIGO Subcommittee. Twenty-five years after the FIGO guidelines for the use of fetal monitoring: time for a simplified approach? Int J Gynaecol Obstet Off Organ Int Fed Gynaecol Obstet 2010;110:1-6.

9. Hruban L, Spilka J, Chudáček V, Janků P, Huptych M, Burša M, Hudec A, Kacerovský M, Koucký M, Procházka M, Korečko V, Seget'a J, Šimetka O, Měchurová A, Lhotská L. Agreement on intrapartum cardiotocogram recordings between expert obstetricians. J Eval Clin Pract 2015;21:694-702.

10. Schifrin BS, Cohen WR. The effect of malpractice claims on the use of caesarean section. Best Pract Res Clin Obstet Gynaecol 2013;27:26983.

11. Cohen WR, Schifrin BS. Medical negligence lawsuits relating to labor and delivery. Clin Perinatol 2007;34:345-60.

12. Siggaard-Andersen $O$, Huch R. The oxygen status of fetal blood. Acta Anaesthesiol Scand 1995;Suppl 107:129-35. 
13. Corn PG. Hypoxic regulation of miR-210: shrinking targets expand HIF-1's influence. Cancer Biol Ther 2008;7:265-7.

14. Wang $H$, Flach $H$, Onizawa M, Wei L, McManus MT, Weiss A. Negative regulation of Hif1a expression and $\mathrm{TH} 17$ differentiation by the hypoxia-regulated microRNA miR-210. Nat Immunol 2014;15:393-401.

15. Gilad S, Meiri E, Yogev $\mathrm{Y}$, Benjamin S, Lebanony $\mathrm{D}$, Yerushalmi $\mathrm{N}$ Benjamin H, Kushnir M, Cholakh H, Melamed N, Bentwich Z, Hod $\mathrm{M}$, Goren $\mathrm{Y}$, Chajut A. Serum microRNAs are promising novel biomarkers. PLoS One 2008;3:e3148.

16. Luo S, Ishibashi O, Ishikawa G, Ishikawa T, Katayama A, Mishima T, Takizawa T, Shigihara T, Goto T, Izumi A, Ohkuchi A, Matsubara S, Takeshita T, Takizawa T. Human villous trophoblasts express and secrete placenta-specific microRNAs into maternal circulation via exosomes. Biol Reprod 2009;81:717-29.

17. Chan YC, Banerjee J, Choi SY, Sen CK. miR-210: the master hypoxamir. Microcirculation 2012;19:215-23.

18. Chan SY, Loscalzo J. MicroRNA-210: a unique and pleiotropic hypoxamir. Cell Cycle Georget Tex 2010;9:1072-83.

19. Whitehead $\mathrm{CL}$, Teh WT, Walker SP, Leung C, Larmour L, Tong S. Circulating MicroRNAs in maternal blood as potential biomarkers for fetal hypoxia in-utero. PLoS One 2013;8:e78487.

20. Flenady V, Koopmans L, Middleton P, Frøen JF, Smith GC, Gibbons K, Coory M, Gordon A, Ellwood D, McIntyre HD, Fretts R, Ezzati M. Major risk factors for stillbirth in high-income countries: a systematic review and meta-analysis. Lancet 2011;377:1331-40.

21. Baggish AL, Hale A, Weiner RB, Lewis GD, Systrom D, Wang F, Wang TJ, Chan SY. Dynamic regulation of circulating microRNA during acute exhaustive exercise and sustained aerobic exercise training. J Physiol 2011;589:3983-94.

22. Jamali Z, Asl Aminabadi N, Attaran R, Pournagiazar F, Ghertasi Oskouei $S$, Ahmadpour F. MicroRNAs as prognostic molecular signatures in human head and neck squamous cell carcinoma: a systematic review and meta-analysis. Oral Oncol 2015;51:321-31.

23. Li G, Zhao A, Péoch M, Cottier M, Mottet N. Detection of urinary cellfree miR-210 as a potential tool of liquid biopsy for clear cell renal cell carcinoma. Urol Oncol 2017;35(5):294-9.

24. Yang W, Ma J, Zhou W, Zhou X, Cao B, Fan D, Hong L. Biological implications and clinical value of mir-210 in gastrointestinal cancer. Expert Rev Gastroenterol Hepatol 2017;1-10.

25. Zhu W, Zhou K, Zha Y, Chen D, He J, Ma H, Liu X1, Le H, Zhang Y. Diagnostic Value of Serum miR-182, miR-183, miR-210, and miR-126 Levels in Patients with Early-Stage Non-Small Cell Lung Cancer. PLoS One 2016;11:e0153046.
26. Lycoudi A, Mavreli D, Mavrou A, Papantoniou N, Kolialexi A. miRNAs in pregnancy-related complications. Expert Rev Mol Diagn 2015;15:999-1010.

27. Munaut C, Tebache L, Blacher S, Noël A, Nisolle M, Chantraine F. Dysregulated circulating miRNAs in preeclampsia. Biomed Rep 2016;5:686-92

28. Chim S, Shing TK, Hung EC, Leung TY, Lau TK, Chiu RW, Lo YM. Detection and characterization of placental microRNAs in maternal plasma. Clin Chem 2008;54:482-90.

29. Morisaki S, Miura K, Higashijima A, Abe S, Miura S, Hasegawa Y Yoshida A, Kaneuchi M, Yoshiura K, Masuzaki H. Effect of labor on plasma concentrations and postpartum clearance of cell-free, pregnancy-associated, placenta-specific microRNAs. Prenat Diagn 2015;35:44-50.

30. Reddy A, Zhong XY, Rusterholz C, Hahn S, Holzgreve W, Redman CW, Sargent IL. The effect of labour and placental separation on the shedding of syncytiotrophoblast microparticles, cell-free DNA and mRNA in normal pregnancy and pre-eclampsia. Placenta 2008;29:942-9.

31. Fasanaro P, D'Alessandra Y, Di Stefano V, Melchionna R, Romani S, Pompilio G, Capogrossi MC, Martelli F. MicroRNA-210 modulates endothelial cell response to hypoxia and inhibits the receptor tyrosine kinase ligand Ephrin-A3. J Biol Chem 2008;283:15878-83.

32. Hunter MP, Ismail N, Zhang X, Aguda BD, Lee EJ, Yu L, Xiao T, Schafer J, Lee ML, Schmittgen TD, Nana-Sinkam SP, Jarjoura D, Marsh CB. Detection of microRNA expression in human peripheral blood microvesicles. PloS One 2008;3:e3694.

33. Vickers KC, Palmisano BT, Shoucri BM, Shamburek RD, Remaley AT. MicroRNAs are transported in plasma and delivered to recipient cells by high-density lipoproteins. Nat Cell Biol 2011;13:423-33.

34. Mouillet JF, Chu T, Hubel CA, Nelson DM, Parks WT, Sadovsky Y. The levels of hypoxia-regulated microRNAs in plasma of pregnant women with fetal growth restriction. Placenta 2010; 31:781-4.

35. Wong L, Lee K, Russell I, Chen C. Endogenous Controls for Real-Time Quantitation of miRNA Using TaqMan MicroRNA Assays. Applied Biosystems Application Note 2010;127:AP11-01.

36. Mouillet JF, Chu T, Sadovsky Y. Expression patterns of placental microRNAs. Birth Defects Res Clin Mol Teratol 2011;91:737-43.

37. Muralimanoharan S, Guo C, Myatt L, Maloyan A. Sexual dimorphism in miR-210 expression and mitochondrial dysfunction in the placenta with maternal obesity. Int J Obes 2015;39:1274-81. 